\title{
Biomassa microbiana, colóides orgânicos e nitrogênio inorgânico durante a vermicompostagem de diferentes substratos
}

\author{
Adriana Maria de Aquino(1), Dejair Lopes de Almeida(1), José Guilherme Marinho Guerra(1) e Helvécio De-Polli(1)
}

(1)Embrapa Agrobiologia, Caixa Postal 74505, CEP 23850-970 Seropédica, RJ. E-mail: adriana@cnpab.embrapa.br, dejair@cnpab.embrapa.br, gmguerra@cnpab.embrapa.br, depolli@cnpab.embrapa.br

\begin{abstract}
Resumo - O objetivo deste trabalho foi avaliar as modificações que ocorrem no substrato durante a vermicompostagem, utilizando-se biomassa microbiana, formas de N mineral, relação C/N e colóides orgânicos totais. As seguintes misturas foram utilizadas como substratos para a vermicompostagem: esterco, esterco + bagaço na proporção de 1:1, e esterco + bagaço + guandu na proporção de 2:1:1. As misturas foram feitas com base em volume e acondicionadas em canteiros de $0,4 \mathrm{~m}^{3}$. Trezentas minhocas da espécie Eisenia foetida e 100 da Eudrilus eugeniae foram introduzidas em cada canteiro. A cada 20 dias, aproximadamente, num total de 126 dias, foram realizadas as amostragens para a realização das análises. O delineamento experimental adotado foi o de blocos ao acaso, com três repetições em esquema fatorial de 3x8 (três substratos e oito épocas de amostragens). As modificações na biomassa microbiana, quociente metabólico e o conteúdo de nitrato e amônio, não possibilitaram caracterizar a época da maturidade dos vermicompostos. O esterco leva cerca de 70 dias para apresentar relação C/N estável, o esterco + bagaço e o esterco + bagaço + guandu levam 90 dias. A determinação dos colóides orgânicos totais é bastante simples, mas não possibilita caracterizar a maturidade dos vermicompostos.
\end{abstract}

Termos para indexação: Oligochaeta, minhocas, vermicomposto, maturidade do composto.

\section{Microbial biomass, organic colloids and inorganic nitrogen, during vermicomposting of some substrates}

\begin{abstract}
The objetive of this work was to evaluate modifications in the microbial biomass, metabolic quotient, types of mineral nitrogen, $\mathrm{C} / \mathrm{N}$ ratio, and total organic colloids concentration. The following variations in the substrate composition were utilized: pure cattle manure, pure cattle manure + sugarcane bagasse 1:1 ratio, and pure cattle manure + sugarcane bagasse + leaves of Cajanus cajan 2:1:1 ratio. The mixture was made on volume bases and the substrate composition filled boxes of $0.4 \mathrm{~m}^{3}$. Three hundred earthworms of the species Eisenia foetida and 100 of Eudrillus eugeniae were introduced in the boxes. Every 20 days, approximately, samples were collected for analysis during a total period of 126 days. The experiment was placed in a randomized complete block design, with a factorial 3x8, with three replications and the following treatments: three types of substrate and eight sampling dates. Microbial biomass, metabolic quotient and types of mineral nitrogen concentrations were not good indexes to characterize the maturity point of the vermicomposting process. Based on $\mathrm{C} / \mathrm{N}$ ratio, the pure cattle manure reached stability in 70 days and sugarcane bagasse and pure cattle manure + sugarcane bagasse + Cajanus cajan leaves in 90 days. The determination of organic colloids was a simple assay, but was not also a good index to characterize the maturity point of the vermicomposting process.
\end{abstract}

Index terms: Oligochaeta, earthworms, vermicompost, compost maturity.

\section{Introdução}

Do ponto de vista da sustentabilidade dos sistemas de produção, a aplicação dos resíduos orgânicos, unicamente como forma de restabelecer o balanço de nutrientes do solo, tornou-se uma função de menor importância. A sincronia da liberação de nutrientes com a necessidade das plantas é uma das metas desejadas (Bünemann et al.,
2004). O grau de decomposição da matéria orgânica, especialmente da que apresenta alta relação $\mathrm{C} / \mathrm{N}$, é muito importante do ponto de vista dos efeitos decorrentes de sua aplicação no solo, já que pode afetar significativamente a produção (Baca et al., 1995), não somente por causa dos efeitos tóxicos, bem conhecidos, como também pela influência do grau de humificação dos compostos orgânicos, na disponibilidade de alguns nutrientes (Wallace, 1994). 
A vermicompostagem do bagaço de cana-de-açúcar, com o esterco bovino, possibilita o reaproveitamento desse resíduo e representa uma alternativa de substituição parcial ao uso do esterco, nas situações em que as quantidades desse material são insuficientes para atender à demanda (Aquino et al., 1994).

O bagaço de cana-de-açúcar contém grande quantidade de constituintes resistentes à decomposição, tais como celulose (50\%), hemicelulose (28\%) e lignina (10\%) (Kewalramani et al., 1988), de forma que a sua decomposição em condições naturais é lenta. $\mathrm{O}$ enriquecimento com leguminosas pode favorecer o processo e melhorar a qualidade dos vermicompostos. Foi observado que a adição de folhas de leucena proporciona produção de vermicomposto mais rico em nitrogênio (Silva, 1992). Essa leguminosa destaca-se entre as demais por seu alto conteúdo de nitrogênio (Aganga \& Tshwenyane, 2003).

Embora a natureza e a composição das substâncias húmicas dos compostos orgânicos seja, provavelmente, o critério mais adequado para determinar o grau de evolução ou maturidade dos compostos orgânicos, sua determinação pelos métodos atuais é onerosa e trabalhosa, o que não permite que seja utilizada em análises rotineiras. Portanto, buscou-se, neste trabalho, método e parâmetro alternativos para a caracterização da maturidade dos vermicompostos que fossem de mais fácil execução. O método avaliado, proposto por Kiehl (1993), para determinação do teor de colóides orgânicos totais, é bastante simples e não dispendioso. Entretanto, não existem resultados disponíveis sobre a aplicação desse método na determinação da maturidade dos vermicompostos.

Em comparação aos parâmetros químicos, as medidas biológicas tendem a ser mais sensíveis às alterações do ambiente. Em decorrência disso, a biomassa microbiana poderia ser uma medida eficaz na indicação da maturidade dos vermicompostos.

O objetivo deste trabalho foi avaliar as modificações que ocorrem em vários substratos, durante a vermicompostagem, utilizando-se a biomassa microbiana e os colóides orgânicos totais para caracterizar a maturidade dos compostos orgânicos, comparados às formas de $\mathrm{N}$ mineral e à relação $\mathrm{C} / \mathrm{N}$ tradicionalmente utilizados.

\section{Material e Métodos}

O experimento foi implantado em junho de 1993, na estação experimental da Embrapa Agrobiologia, no Município de Seropédica, RJ. Foram utilizados esterco bovino, bagaço de cana-de-açúcar e folhas de guandu como substratos para a vermicompostagem (Tabela 1). A matéria orgânica total foi determinada de acordo com Brasil (1983); o C total foi obtido indiretamente, pela queima em mufla; o $\mathrm{N}$ total foi obtido conforme Bremner (1965), e o P total, de acordo com Bataglia et al. (1983).

O esterco bovino, procedente de rebanho leiteiro da Pesagro (criado em pasto), foi recolhido fresco e, cerca de 15 dias depois, quando a temperatura já se encontrava estabilizada, foi utilizado no experimento. $\mathrm{O}$ bagaço de cana-de-açúcar fresco, procedente de pequeno comércio de caldo de cana localizado nas imediações da Embrapa Agrobiologia, foi picado em picadeira para forragens, em fragmentos de $3-4 \mathrm{~cm}$. As folhas de guandu (Cajanus cajans (L.) Millsp.) foram obtidas de arbustos do campo experimental da Embrapa Agrobiologia.

Os materiais orgânicos foram colocados em canteiros de bambu de $0,4 \mathrm{~m}^{3}$ ( $\left.1 \times 1 \times 0,4 \mathrm{~m}\right)$, num total de nove canteiros. Cada três canteiros continham os seguintes substratos: esterco (E), esterco + bagaço (EB) na proporção de 1:1, e esterco + bagaço + guandu (EBG) na proporção de 2:1:1. As misturas foram feitas com base em volume, num total de $0,35 \mathrm{~m}^{3}$ por canteiro, tendo ocupado $0,35 \mathrm{~m}$ de altura e o restante preenchido com palha seca obtida de grama batatais, a fim de manter a umidade dos materiais orgânicos. Os canteiros foram cobertos com uma estrutura de sapé, para evitar o impacto da chuva.

Depois de sete dias da mistura dos materiais orgânicos, foram introduzidas 400 minhocas adultas em cada canteiro, das quais 300 da espécie Eisenia foetida, e 100 da Eudrilus eugeniae, vulgarmente conhecidas como vermelha-da-califórnia e noturna africana, com peso médio de 0,3 e 1,5 g (peso de matéria fresca), respectivamente.

Foram realizadas oito amostragens, inclusive a inicial (0, 26, 49, 68, 77, 89, 105 e 126 dias, depois da incubação), para as análises descritas a seguir. A amostragem inicial foi realizada após o período de sete dias da incubação, porém antes da introdução das minhocas.

Tabela 1. Caracterização química de esterco bovino, bagaço de cana-de-açúcar e guandu.

\begin{tabular}{|c|c|c|c|}
\hline Parâmetro $^{(1)}$ & Esterco & Bagaço & Guandu \\
\hline & \multicolumn{3}{|c|}{ - $\left(\mathrm{g} \mathrm{kg}^{-1}\right)$} \\
\hline Umidade a $65^{\circ} \mathrm{C}$ & 643 & 613 & 587 \\
\hline Carbono & 398 & 546 & 523 \\
\hline Nitrogênio & 12 & 2 & 31 \\
\hline Relação C/N & 33 & 273 & 17 \\
\hline Matéria orgânica & 717 & 983 & 942 \\
\hline Fósforo & 4 & 10 & 5 \\
\hline Potássio & 15 & 90 & 24 \\
\hline
\end{tabular}

${ }^{(1)} \mathrm{Em}$ base seca a $65^{\circ} \mathrm{C}$. 
A estimativa da biomassa microbiana foi feita pelo método da fumigação-extração, descrito por De-Polli \& Guerra (1999), com a relação amostra:extrator modificada de 1:2,5 para 1:10. A atividade respiratória microbiana basal foi obtida pela incubação das amostras das misturas dos substratos, com retenção de $\mathrm{CO}_{2}$ por $\mathrm{NaOH}$ por sete dias. A taxa de respiração específica ou quociente metabólico, designada como $q \mathrm{CO}_{2}$, foi calculada como a taxa de produção de $\mathrm{C}\left(\right.$ do $\left.\mathrm{CO}_{2}\right)$ por determinada quantidade de $\mathrm{C}$ microbiano em função do tempo.

A análise de $\mathrm{C}$ foi feita pelo método descrito em Brasil (1983), e os teores de $\mathrm{N}$ total, nítrico e amoniacal foram obtidos pela extração com $\mathrm{KCl}$ na relação extrator:amostra de 2:1, conforme descrito por Bremner (1965).

Os colóides orgânicos e conteúdo de matéria orgânica humificada, foram determinados de acordo com a metodologia descrita por Kiehl (1993). Nessa determinação, foram utilizados $20 \mathrm{~g}$ de amostra, $50 \mathrm{~mL}$ de álcool etílico e $400 \mathrm{~mL}$ de $\mathrm{H}_{2} \mathrm{O}$ destilada. Após reação alcalina com $\mathrm{NaOH}$ 0,5 N, a amostra foi aquecida até ebulição. Depois do resfriamento, essa mistura foi lavada em peneira de 0,053 mm numa proveta de $1.000 \mathrm{~mL}$ e, após completar o volume, o líquido da proveta foi agitado. Introduziu-se uma pipeta de $20 \mathrm{~mL}$ à profundidade de $10 \mathrm{~cm}$, e retirou-se uma alíquota que foi levada à estufa a $100-105^{\circ} \mathrm{C}$ e depois para a mufla a $550^{\circ} \mathrm{C}$. A quantidade de colóides totais na amostra a $20 \mathrm{~g}$ foi obtida pela subtração dos pesos após estufa e mufla, tendo-se corrigido os valores com base no teor de matéria orgânica da amostra.

O delineamento experimental adotado foi o de blocos ao acaso com três repetições, em esquema fatorial de 3x8, constituído de três substratos e oito épocas de amostragens.

As épocas de amostragens foram consideradas como subparcelas dos substratos, quando as variâncias eram homogêneas. De acordo com o teste de Bartlett, as seguintes variáveis apresentaram variâncias homogêneas: relação $\mathrm{C} / \mathrm{N}$ e colóides orgânicos totais. De acordo com esse teste, as variáveis biomassa microbiana, quociente metabólico, conteúdo de nitrato e amônio apresentaram variâncias não homogêneas. Por isso, para esses parâmetros, a análise de variância foi realizada por coleta. Utilizou-se o teste de Tukey a 5\% de probabilidade, para realizar a discriminação das médias. Foram realizadas análises de correlações entre os diferentes parâmetros, e apresentadas as equações de regressão para as correlações significativas.

\section{Resultados e Discussão}

Aos 26 dias após a vermicompostagem, o substrato EB apresentou maior valor de biomassa microbiana, possivelmente pela maior oferta de $\mathrm{C}$ e energia para utilização dos microrganismos (Tabela 2). No entanto, apresentou menor valor de $q \mathrm{CO}_{2}$ na mesma época, o que significa que, embora nessas condições os microrganismos dispusessem de $\mathrm{C}$ e energia para se multiplicarem, a eficiência de utilização desse $C$ foi baixa, ou seja, existem muitos microrganismos, mas com baixa atividade.

A vermicompostagem leva ao declínio da população das minhocas adultas (Aquino et al., 1994), seja pelo estresse provocado pela alta densidade populacional (Ndegwa et al., 2000), causada pela reprodução e aumento da população das minhocas jovens, seja pela redução da oferta de alimento, proveniente de matéria orgânica já estabilizada. Embora não tenha sido detectada a influência dessa mortalidade em diversos parâmetros químicos, como em Aquino (1991) ou em Silva (1992), os aumentos dos valores da biomassa microbiana, observados entre cerca de 70 e 90 dias e após 100 dias (Tabela 2), provavelmente foram decorrentes desse efeito, uma vez que o tecido das minhocas

Tabela 2. Valores da biomassa microbiana $\left(\mu \mathrm{g} \mathrm{C} \mathrm{g}{ }^{-1}\right)$ e $q \mathrm{CO}_{2}\left(\mu g \mathrm{C}^{-} \mathrm{CO}_{2} \mathrm{cg}^{-1} \mathrm{C}_{\mathrm{BM}}\right.$ h) durante a vermicompostagem de substratos com esterco (E), esterco + bagaço (EB) e esterco + bagaço + guandu (EBG) ${ }^{(1)}$.

\begin{tabular}{|c|c|c|c|c|c|c|c|c|}
\hline \multirow[t]{2}{*}{ Tempo $^{(2)}$} & \multicolumn{4}{|c|}{ Biomassa microbiana } & \multicolumn{4}{|c|}{$q \mathrm{CO}_{2}$} \\
\hline & $\mathrm{E}$ & EB & EBG & CV (\%) & E & EB & EBG & CV (\%) \\
\hline 0 & $13.829 a$ & $23.107 a$ & 17.119a & 31 & $1.250 \mathrm{a}$ & $1.210 \mathrm{a}$ & $1.360 \mathrm{a}$ & 10 \\
\hline 26 & $7.906 \mathrm{~b}$ & $14.154 \mathrm{a}$ & $7.705 \mathrm{~b}$ & 8 & $4.310 \mathrm{a}$ & $2.600 \mathrm{~b}$ & $4.940 \mathrm{a}$ & 12 \\
\hline 49 & $4.332 b$ & $7.659 \mathrm{a}$ & $6.853 a$ & 4 & $6.410 \mathrm{a}$ & $4.440 \mathrm{~b}$ & $5.200 \mathrm{~b}$ & 7 \\
\hline 68 & $2.099 \mathrm{a}$ & $2.992 \mathrm{a}$ & $3.769 \mathrm{a}$ & 43 & $9.360 \mathrm{a}$ & $8.640 \mathrm{a}$ & $9.540 \mathrm{a}$ & 51 \\
\hline 77 & $5.313 b$ & $7.960 \mathrm{a}$ & $8.456 \mathrm{a}$ & 12 & $3.110 \mathrm{a}$ & $3.070 \mathrm{a}$ & $2.930 \mathrm{a}$ & 14 \\
\hline 89 & $4.432 \mathrm{a}$ & $8.871 \mathrm{a}$ & $7.270 \mathrm{a}$ & 53 & $3.420 \mathrm{a}$ & $3.040 \mathrm{a}$ & $7.980 \mathrm{a}$ & 101 \\
\hline 105 & $1.199 \mathrm{a}$ & $1.261 \mathrm{a}$ & $1.209 \mathrm{a}$ & 35 & $27.300 \mathrm{a}$ & $36.700 \mathrm{a}$ & $41.800 \mathrm{a}$ & 38 \\
\hline 126 & $5.555 \mathrm{a}$ & $7.724 \mathrm{a}$ & $6.857 \mathrm{a}$ & 24 & $2.700 \mathrm{a}$ & $2.970 \mathrm{a}$ & $2.630 \mathrm{a}$ & 21 \\
\hline
\end{tabular}

${ }^{(1)}$ Valores seguidos de letras iguais, na linha, não diferem entre si a 5\% de probabilidade pelo teste de Tukey. (2)Dias após a vermicompostagem. 
mortas representa adição de matéria orgânica fresca facilmente acessível aos microrganismos, especialmente da comunidade zimogênica. Como o "turnover" de C e $\mathrm{N}$ na biomassa microbiana é muito rápido, a medida da biomassa pode refletir as alterações do meio, antes que essas possam ser detectadas por $\mathrm{C}$ e $\mathrm{N}$ total, por exemplo. Bending et al. (2004) afirmaram que a biomassa microbiana pode ser um parâmetro interessante como bioindicador de manejo.

A biomassa microbiana é uma medida que expressa a quantidade de microrganismos vivos presentes no substrato, mas não reflete a atividade deles. $\mathrm{O}_{\mathrm{qCO}}$, por sua vez, pode ser um parâmetro complementar, pois trata a biomassa microbiana coletivamente como uma reação ao substrato disponível.

Embora a biomassa microbiana, no início do processo de decomposição, tenha sido alta (Tabela 2), os valores de $q \mathrm{CO}_{2}$ tenderam a ser mais baixos, comparados com os demais valores obtidos no decorrer do processo, o que pode indicar predomínio da comunidade microbiana autóctone e caracterizar baixa atividade microbiana.

O bagaço de cana-de-açúcar puro apresenta uma relação C/N muito alta, de aproximadamente 200 (Tabela 1), com grande quantidade de constituintes resistentes à decomposição, tais como celulose (50\%), hemicelulose (28\%) e lignina (10\%) (Kewalramani et al., 1988). A decomposição limitada impossibilita a sobrevivência das minhocas nesse substrato (Aquino et al., 1994).

Quando o bagaço foi misturado ao esterco bovino na proporção de 1:1 (v/v), a relação $\mathrm{C} / \mathrm{N}$ inicial caiu para 36 (Tabela 3), aproximadamente seis vezes menor que a relação $\mathrm{C} / \mathrm{N}$ do bagaço puro. O esterco possivelmente funciona como fonte de microrganismos e de $\mathrm{N}$, bem como possibilita a sobrevivência e reprodução das minhocas (Aquino et al., 1994). O bagaço misturado ao esterco torna-se mais facilmente decomponível, oferece suprimento de energia e favorece o aumento da popula-

Tabela 3. Relação C/N durante a vermicompostagem de substratos com esterco (E), esterco + bagaço (EB) e esterco + bagaço + guandu $(\mathrm{EBG})^{(1)}$.

\begin{tabular}{cccl}
\hline Tempo $^{(2)}$ & $\mathrm{E}$ & $\mathrm{EB}$ & $\mathrm{EBG}$ \\
\hline 0 & $23 \mathrm{Ac}$ & $36 \mathrm{Aa}$ & $26 \mathrm{Ab}$ \\
26 & $20 \mathrm{Bb}$ & $26 \mathrm{Ba}$ & $22 \mathrm{Bb}$ \\
49 & $20 \mathrm{Bb}$ & $24 \mathrm{Ba}$ & $18 \mathrm{Cb}$ \\
68 & $17 \mathrm{Cb}$ & $24 \mathrm{Ba}$ & $18 \mathrm{Cb}$ \\
77 & $16 \mathrm{Cb}$ & $25 \mathrm{Ba}$ & $18 \mathrm{Cb}$ \\
89 & $15 \mathrm{Cb}$ & $20 \mathrm{Ca}$ & $16 \mathrm{CDb}$ \\
105 & $16 \mathrm{Cb}$ & $19 \mathrm{Ca}$ & $16 \mathrm{CDb}$ \\
126 & $16 \mathrm{Ca}$ & $18 \mathrm{Ca}$ & $15 \mathrm{Da}$ \\
\hline
\end{tabular}

(1)Valores seguidos de letras iguais, maiúsculas na coluna e minúsculas na linha, não diferem entre si a 5\% de probabilidade pelo teste de Tukey; o coeficiente de variação foi 8\%. (2)Dias após a vermicompostagem. ção de microrganismos. À medida que os microrganismos e as minhocas vão consumindo o $\mathrm{C}$, e liberando $\mathrm{CO}_{2}$, a relação $\mathrm{C} / \mathrm{N}$ diminui.

A adição de minhocas nesse processo pode promover a obtenção de um produto final com mais baixa relação $\mathrm{C} / \mathrm{N}$, maior capacidade de troca catiônica e maior quantidade de substâncias húmicas (Orozco et al., 1996).

Embora a relação $\mathrm{C} / \mathrm{N}$ não possa ser utilizada como indicador absoluto da maturação do composto, pois depende do material original, é um dos índices mais utilizados, e define, inclusive, sua qualidade agronômica (Berna et al., 1996).

A estabilização da relação $\mathrm{C} / \mathrm{N}$ foi observada em torno de 70 dias para E e 90 dias para EB e EBG (Tabela 3). Embora a estabilização tenha ocorrido na mesma época, a relação C/N de EBG foi significativamente menor que a de EB (Tabela 3), o que indica que a adição de material mais rico em $\mathrm{N}$, durante a vermicompostagem, pode promover mais baixa relação $\mathrm{C} / \mathrm{N}$, conforme constatado por Silva (1992), com a adição de leucena ao processo.

De uma maneira geral, o composto com a relação $\mathrm{C} / \mathrm{N}$ abaixo de 20 é considerado relativamente estável (Berna et al., 1996). Os valores obtidos da relação C/N, para os vermicompostos considerados estabilizados, estão de acordo com o referido por esses autores, para compostos produzidos sem minhocas.

A mineralização de N é definida como a transformação do N orgânico em formas inorgânicas. A partir desse processo, os organismos heterotróficos utilizam substâncias orgânicas nitrogenadas como fonte de energia (Jansson \& Persson, 1982). A mineralização envolve os processos de amonificação e nitrificação (Cantarella et al., 1992).

Observou-se constante liberação de amônio com a vermicompostagem, o que indica a ocorrência do processo de amonificação independentemente do substrato (Tabela 4). O amônio é considerado um subproduto do metabolismo microbiano, que é liberado quando sua quantidade excede a demanda das células. Já aos 68 dias de amostragem, E apresentou conteúdo dessa forma de $\mathrm{N}$ significantemente menor do que EB e EBG. O decréscimo de amônio foi acompanhado, como esperado, pelo processo de nitrificação, no qual foi oxidado a nitrato, indicado pelo significante conteúdo desse nutriente em E a partir dos 68 dias (Tabela 4).

$\mathrm{O}$ aumento de nitrato tem sido observado ao longo do processo de compostagem (Paré et al., 1998), em razão da atividade das bactérias nitrificadoras aumentar com a bioestabilidade dos resíduos orgânicos; por isso, o acúmulo de nitrato tem sido utilizado como indicador da maturidade do processo de compostagem. 
Tem sido observado que as minhocas promovem, durante a vermicompostagem, redução do teor de amônio (Elvira et al., 1998). A nitrificação pode ser otimizada pela atividade das minhocas, que promovem melhores condições aeróbicas para os microrganismos por meio das galerias que criam, ou pelo aumento da área superficial do substrato, após a ingestão deste (Parkin \& Berry, 1999).

A imobilização de $\mathrm{N}$ é a transformação de compostos inorgânicos de $\mathrm{N}\left(\mathrm{NH}_{4}{ }^{+}, \mathrm{NH}_{3}, \mathrm{NO}_{3}{ }^{-}\right.$e $\left.\mathrm{NO}_{2}{ }^{-}\right)$em formas orgânicas, em que os organismos assimilam o $\mathrm{N}$ inorgânico e o transformam em constituintes orgânicos de $\mathrm{N}$ de suas células e tecidos (Jansson \& Persson, 1982). Assim, em toda atividade de mineralização há um componente de imobilização, uma renovação da matéria orgânica e uma assimilação de nutrientes minerais, que promovem crescimento e manutenção da biomassa. O efeito dos dois processos, expresso como mineralização líquida ou imobilização líquida é determinado pelo suprimento de N. Comparando-se os substratos, verifica-se que o processo de nitrificação foi diferenciado somente aos 68 e 89 dias (Tabela 4). É possível que a eficiência de assimilação de $\mathrm{C}$ pelas minhocas e, conseqüentemente, o estímulo à atividade microbiana em E, seja maior que em EB e EBG, o que foi evidenciado pelo menor conteúdo de amônio aos 68 dias e maior de nitrato aos 68 e 89 dias.

De modo geral, as variações das formas de $\mathrm{N}$ não seguiram um padrão muito bem definido, tendo dificultado a interpretação da nitrificação durante o processo da vermicompostagem. Como referido anteriormente, o processo de vermicompostagem pode levar à morte das minhocas. As minhocas mortas decompõem-se muito rapidamente, liberando aproximadamente $12 \%$ de $\mathrm{N}$ (Barley, 1965), o que pode levar a algumas variações das formas inorgânicas de $\mathrm{N}$ durante a vermicompostagem.
Os colóides orgânicos totais se referem ao conteúdo de matéria orgânica humificada (Kiehl, 1993). A humificação é um processo que, possivelmente, depende do balanço entre mineralização, policondensação e neossíntese (Stevenson, 1982).

A fração coloidal inclui ácido húmico, ácido fúlvico, ácido himatomelânico, ácido apocrênico, humina, enfim todos os compostos que se encontram na forma coloidal (Kiehl, 1993). Os materiais orgânicos não decompostos têm características diferentes da matéria orgânica estabilizada, que influi nas propriedades físicas e químicas do solo. Assim, o valor de um fertilizante orgânico, como condicionador do solo, pode ser avaliado em função do conteúdo de matéria orgânica humificada.

No início do experimento, o teor de colóides totais foi maior em E (Tabela 4). O esterco diferiu do bagaço, entre outros, por ter sofrido transformações precedentes no trato digestivo do bovino. Em conseqüência disso, o esterco fresco apresentou uma quantidade maior de frações humificadas, porém, possivelmente com moléculas pouco polimerizadas e de baixo peso molecular.

Tem sido demonstrado que o processo de vermicompostagem leva ao aumento do conteúdo das substâncias húmicas (Elvira et al., 1998). Esperava-se, conseqüentemente, que a quantidade de colóides totais aumentasse com o tempo até a estabilização. No entanto, os colóides totais estabeleceram relação linear com o tempo em todos os substratos, não tendo apresentado a estabilização esperada. Com base nos resultados da análise de variância da regressão, foi constatada regressão linear para E $\left(r^{2}=0,80^{* *}\right)$, EB $\left(r^{2}=0,67^{* *}\right)$ e EBG $\left(r^{2}=0,75^{* *}\right)$.

Apesar de os resultados não terem indicado estabilização dos colóides totais em nenhum dos substratos, estes se correlacionaram significativamente com outros parâmetros (Tabela 5) que, conhecidamente, se relacionam com a maturidade dos compostos, inclusive a relação

Tabela 4. Conteúdo de nitrogênio mineral $\left(\mathrm{mg} \mathrm{kg}^{-1}\right)$ e teor de colóides orgânicos totais (\%), em relação à matéria orgânica total, durante a vermicompostagem de substratos com esterco (E), esterco + bagaço (EB) e esterco + bagaço + guandu (EBG) ${ }^{(1)}$.

\begin{tabular}{|c|c|c|c|c|c|c|c|c|c|c|c|}
\hline \multirow[t]{2}{*}{ Tempo $^{(2)}$} & \multicolumn{4}{|c|}{$\mathrm{NH}_{4}^{+}$} & \multicolumn{4}{|c|}{$\mathrm{NO}_{3}{ }^{-}$} & \multicolumn{3}{|c|}{ Colóides totais } \\
\hline & $\mathrm{E}^{(1)}$ & EB & EBG & CV (\%) & $\mathrm{E}$ & EB & EBG & CV (\%) & $\mathrm{E}$ & EB & EBG \\
\hline 0 & $40,6 a$ & $47,5 \mathrm{a}$ & $67,5 \mathrm{a}$ & 39 & $4,8 \mathrm{a}$ & $8,1 \mathrm{a}$ & $5,5 \mathrm{a}$ & 62 & $38 \mathrm{a}$ & $17 \mathrm{~b}$ & $27 a b$ \\
\hline 26 & $55,8 \mathrm{a}$ & $46,6 a$ & $73,1 \mathrm{a}$ & 34 & $19,9 \mathrm{a}$ & $8,1 \mathrm{a}$ & $32,1 \mathrm{a}$ & 72 & $51 \mathrm{a}$ & $31 b$ & $41 \mathrm{ab}$ \\
\hline 49 & $108,0 \mathrm{a}$ & $168,9 \mathrm{a}$ & $207,5 \mathrm{a}$ & 15 & $81,3 a$ & $5,8 \mathrm{a}$ & $29,5 \mathrm{a}$ & 133 & $41 \mathrm{a}$ & $54 a$ & $50 \mathrm{a}$ \\
\hline 68 & $18,1 \mathrm{~b}$ & $37,5 \mathrm{a}$ & $49,7 \mathrm{a}$ & 23 & $454,3 \mathrm{a}$ & $8,4 \mathrm{~b}$ & $160,8 \mathrm{ab}$ & 56 & $43 a$ & $43 a$ & $42 a$ \\
\hline 77 & $49,5 \mathrm{a}$ & $75,9 a$ & $70,0 \mathrm{a}$ & 33 & $604,7 \mathrm{a}$ & $19,0 \mathrm{a}$ & $348,6 a$ & 65 & $53 a$ & $38 b$ & $38 b$ \\
\hline 89 & $26,6 \mathrm{a}$ & $50,5 \mathrm{a}$ & $45,7 \mathrm{a}$ & 29 & $668,6 a$ & $11,7 \mathrm{~b}$ & $168,1 \mathrm{~b}$ & 41 & $46 a$ & $47 \mathrm{a}$ & $56 a$ \\
\hline 105 & $25,4 \mathrm{a}$ & $48,3 \mathrm{a}$ & $35,1 \mathrm{a}$ & 33 & $575,5 \mathrm{a}$ & $80,5 \mathrm{a}$ & $525,7 \mathrm{a}$ & 63 & $65 a$ & $55 \mathrm{a}$ & $60 \mathrm{a}$ \\
\hline 126 & $36,8 \mathrm{a}$ & $63,0 \mathrm{a}$ & $58,6 \mathrm{a}$ & 21 & $552,4 \mathrm{a}$ & $278,5 \mathrm{a}$ & $406,5 \mathrm{a}$ & 65 & $67 \mathrm{a}$ & $62 \mathrm{a}$ & $70 \mathrm{a}$ \\
\hline
\end{tabular}

${ }^{(1)}$ Valores seguidos de letras iguais, na linha, não diferem entre si a 5\% de probabilidade pelo teste de Tukey; o coeficiente de variação dos dados de colóides totais foi $12 \%$. ${ }^{(2)}$ Dias após a vermicompostagem. 
Tabela 5. Coeficientes de correlação linear (r) entre colóides totais (\%) dos vermicompostos e diferentes parâmetros.

\begin{tabular}{lcc}
\hline Variáveis independentes & Equação de regressão & Coeficiente de correlação (r) \\
\hline Nitrogênio (\%) & $\mathrm{y}=-7,53+34,90 \mathrm{x}$ & $0,49^{* * *}$ \\
Carbono (\%) & $\mathrm{y}=129,61-2,62 \mathrm{x}$ & $-0,79^{* * *}$ \\
Relação C/N & $\mathrm{y}=90,27-2,10 \mathrm{x}$ & $-0,75^{* * *}$ \\
$\mathrm{NO}_{3}{ }^{-}\left(\mathrm{mg} \mathrm{kg}{ }^{-1}\right)$ & $\mathrm{y}=45,25+0,026 \mathrm{x}$ & $0,50^{* * *}$ \\
Biomassa microbiana $\left(\mu \mathrm{g} \mathrm{C} \mathrm{g}^{-1}\right)$ & $\mathrm{y}=58,06-0,001 \mathrm{x}$ & $-0,59^{* * *}$ \\
\hline
\end{tabular}
$* * * \mathrm{p}<0,0001$.

C/N, um dos parâmetros mais utilizados (Berna et al., 1996; Salamanca et al., 2003).

\section{Conclusões}

1. Os parâmetros microbiológicos, biomassa microbiana e quociente metabólico, utilizados para avaliar a dinâmica da vermicompostagem, bem como o conteúdo de amônio e nitrato, indicam variações no decorrer do processo, mas não possibilitam caracterizar a época da maturidade dos vermicompostos.

2. Os valores de colóides orgânicos totais têm uma relação linear com o tempo em todos os substratos e não apresentam a estabilização esperada.

3. Os colóides orgânicos totais se correlacionam significativa e negativamente com a relação $\mathrm{C} / \mathrm{N}$, e o processo de vermicompostagem deve se estender por mais tempo para possível estabilização.

\section{Referências}

AGANGA, A.A.; TSHWENYANE, S.O. Lucerne, Lablab and Leucaena leucocephala forages: production and utilization for livestock production. Pakistan Journal of Nutrition, v.2, p.4653, 2003.

AQUINO, A.M. de; ALMEIDA, D.L. de; FREIRE, L.R.; DE-POLLI, H. Reprodução de minhocas (Oligochaeta) em esterco bovino e bagaço de cana-de-açúcar. Pesquisa Agropecuária Brasileira, v.29, p.161168, 1994.

AQUINO, V.F. da. Vermicompostagem de esterco bovino e bagaço de cana-de-açúcar inoculados com a bactéria fixadora de $\mathbf{N}_{2}$ (Acetobacter diazotrophicus). 1991. 246p. Dissertação (Mestrado) - Universidade Federal Rural do Rio de Janeiro, Seropédica.

BACA, M.T.; DELGADO, I.C.; DE NOBILI, M.; ESTEBAN, E.; SANCHEZ-RAYA, A.J. Influence of compost maturity on nutrient status of sunflower. Comunications in Soil Science and Plant Analysis, v.26, p.169-181, 1995.

BARLEY, K.P. The abundance of earthworms in agricultural land and their possible significance in agriculture. Advances in Agronomy, v.13, p.249-268, 1961.

BATAGLIA, O.C.; FURLANI, A.M.C.; TEIXEIRA, J.P.F.; FURLANI, P.R.; GALLO, J.R. Métodos de análise química de plantas. Boletim Técnico do Instituto Agronômico, v.78, p.1-48, 1983.
BENDING, G.D.; TURNER, M.K.; RAYNS, F.; MARX, M.; WOOD, M. Microbial and biochemical soil quality indicators and their potential for differentiating areas under contrasting agricultural management regimes. Soil Biology and Biochemistry, v.36, p.17851792, 2004.

BERNA, M.P.; NAVARRO, A.F.; ROIG, A.; CEGARRA, J.; GARCÍA, D. Carbon and nitrogen transformation during composting of sweet sorghum bagasse. Biology and Fertility of Soils, v.22, p.141-148, 1996.

BRASIL. Ministério da Agricultura. Secretaria Nacional de Referência Vegetal. Análise de corretivos, fertilizantes e inoculantes: métodos oficiais. Brasília, 1983. 104p.

BREMNER, J.M. Total nitrogen; Inorganic forms of nitrogen. In: BLACK, C.A.; EVANS, D.D.; WHITE, J.L.; ENSMINGER, L.E.; CLARK, F.E. (Ed.). Methods of soil analysis: part 2. Madison: American Society of Agronomy, 1965. p.1149-1255. (Agronomy, 9).

BÜNEMANN, E.K.; BOSSIO, D.A.; SMITHSON, P.C.; FROSSARD, E.; OBERSON, A. Microbial community composition and substrate use in a highly weathered soil as affected by crop rotation and P fertilization. Soil Biology and Biochemistry, v.36, p.889-901, 2004.

CANTARELLA, H.; ABREU, C.A. de; BERTON, R.S. Fornecimento de nutrientes pela matéria orgânica do solo. In: ENCONTRO SOBRE MATÉRIA ORGÂNICA DO SOLO - PROBLEMAS E SOLUÇÕES, 1992, Botucatu. Anais. Botucatu: Faculdade de Ciências Agronômicas, 1992. Editado por I. A. Guerrini, L. T. Bull. p.63-122.

DE-POLLI, H.; GUERRA, J.G.M. C, N e P na biomassa microbiana do solo. In: SANTOS, G.A.; CAMARGO, F.A.O. (Ed.). Fundamentos da matéria orgânica do solo: ecossistemas tropicais e subtropicais. Porto Alegre: Genesis, 1999. p.389-411.

ELVIRA, C.; SAMPEDRO, L.; BENÍTEZ, E.; NOGALES, R. Vermicomposting of sludges from paper mill and dairy industries with Eisenia andrei: a pilot-scale study. Bioresource Technology, v.63, p.205-211, 1998.

JANSSON, S.L.; PERSSON, J. Mineralization and immobilization of soil nitrogen. In: STEVENSON, F.J. (Ed.). Nitrogen in agricultural soils. Madison: American Society of Agronomy, 1982. p.229-252. (Agronomy, 22).

KEWALRAMANI, N.; KAMRA, D.N.; LALL, D.; PATHAK, N.N. Bioconversion of sugarcane bagasse with white rot fungi. Biotechnology Letters, v.10, p.369-372, 1988.

KIEHL, E.J. Fertilizantes organominerais. Piracicaba: USP, 1993. 189p. 
MYERS, R.J.K.; PALM, C.A.; CUEVAS, E.; GUNATILLEKE, I.U.N.; BROSSARD, M. The synchronization of nutrient mineralization and plant nutrient demand. In: WOOMER, P.L.; SWIFT, M.J. (Ed.). The biological management of tropical soil fertility. New York: J. Wiley, 1994. p.81-116.

NDEGWA, P.M.; THOMPSON, S.A.; DAS, K.C. Effects of stocking density and feeding rate on vermicomposting of biosolids. Bioresource Technology, v.71, p.5-12, 2000.

OROZCO, F.H.; CEGARRA, J.; TRUJILLO, L.M.; ROIG, A. Vermicomposting of coffee pulp using the earthworm Eisenia foetida: effects on $\mathrm{C}$ and $\mathrm{N}$ contents and the availability of nutrients. Biology and Fertility of Soils, v.22, p.162-166, 1996.

PARÉ, T.; DINEL, H.; SCHNITZER, M.; DUMONTET, S. Transformations of carbon and nitrogen during composting of animal manure and shredded paper. Biology and Fertility of Soils, v.26, p.173-178, 1998.
PARKIN, T.B.; BERRY, E.C. Microbial nitrogen transformations in earthworm burrows. Soil Biology and Biochemistry, v.31, p.17651771, 1999.

SALAMANCA, E.F.; KANEKO, N.; KATAGIRI, S. Rainfall manipulation effects on litter decomposition and the microbial biomass of the forest floor. Applied Soil Ecology, v.22, p.271-281, 2003.

SILVA, V.F. da. Vermicompostagem utilizando esterco e palha enriquecida com $\mathbf{N}$ e $\mathbf{P}$ : processo de produção e avaliação para a cultura da cenoura (Daucus carota L.). 1992. 118p. Dissertação (Mestrado) - Universidade Federal Rural do Rio de Janeiro, Seropédica.

STEVENSON, F.J. Humus chemistry. Mississauga, Canada: J. Wiley \& Sons, 1982. 443p.

WALLACE, A. Soil organic matter is essential to solving soil and environmental problems. Communications in Soil Science and Plant Analysis, v.25, p.15-28, 1994.

Recebido em 2 de abril de 2004 e aprovado em 4 de maio de 2005 